\title{
A Multimedia Teaching System with Special Acoustic Effects in the Course of Aerobics
}

\author{
https://doi.org/10.3991/ijet.v11i10.6266 \\ Yingbao Zhou \\ Nanjing Xiaozhuang University, Nanjing, Jiangsu, China
}

\begin{abstract}
With the continuous advancement of multimedia technology, multimedia teaching has also posed higher requirements on multimedia playing capabilities. This essay presents the design program for a speaker which could be applied in multimedia classroom to achieve special acoustic effects based on use of the ASP.NET technology. The SQL Server 2005 database technology and Microsoft Visual Studio 2008 technology are combined in the design process. Compared with traditional multimedia speakers, the speak of this program is small, portable, and power-saving, may bring changeable lighting stage effects, could be easily connected to computers, and support playing files stored in $U$ disk and SD card. After the entire program is practically utilized in teaching, it has been found that the system can meet acoustic demand of teachers and students, and improve teaching effectiveness.
\end{abstract}

Index Terms - special acoustic effects, multimedia teaching system, application in courses

\section{INTRODUCTION}

With the advent of the information age and the improvement of technological levels, the traditional teaching method cannot meet the present educational needs, explaining why audio-visual education has become increasingly popular [1]. Currently, the most common classroom in audio-visual education is multimedia classroom, a modern classroom equipped with multimedia computer, LCD projector, digital video display, the central control system, projection screen, audio equipment, and other devices [2]. Multimedia classroom can demonstrate videos, audios, software presentations to students, mobilize their enthusiasm, and enable them to acquire a deeper understanding of knowledge through visual, auditory and manipulative learning [3]. For the delivery of educational content in dentistry, Salajan et al. presented the development and implementation of Dental Procedure Education System, a new Web and wiki-based application which integrates Share Point with wiki-based space technology to use multiple functions of Wiki. The corresponding experimental result shows that this new multimedia teaching can bring students an authoritative source of knowledge and information and contribute to higher teaching effectiveness [4]. Meanwhile, a research team has developed a new interactive multimedia classroom learning system, in which Q\&A, whiteboard, video conferencing and VoIP are included. In this system, the user can learn via the Web network system, or engage in interactive learning activities via video and VoIP. This new multimedia teaching system has been recognized by many users [5]. At the same time, studies have shown that the modern multimedia teaching system is also suitable for physical education, because the dynamic process of presenting pictures by multimedia technology can increase the intuitiveness and fun in various teaching links. Compared with the previous single way of outdoor teaching, students tend to select the learning approach which combines multimedia teaching and outdoor classroom [6].

Aerobics, gymnastics, dance and similar courses focus on physical education related to students' body language [7]. Therefore, teaching of these courses requires a combination of movement and music. For example, audio equipment should be used to strengthen the performance results, create a specific environment and highlight the theme. After analyzing the effect of tone tuning in live performances, Li (2014) considered acoustics as one of the important props to heighten the stage atmosphere and to achieve special intervention effects on physical performance [8].

Although the function and role of multimedia classroom audio equipment are obvious, many schools now are using multimedia classrooms converted from traditional classrooms. During the process of constructing multimedia classrooms, attention has often been paid only to the quality of the computer, whether the speaker is big, and whether the projection is clear. This major drawback is mainly reflected in the design of acoustic environment of multimedia classrooms. According to the survey, most of the multimedia classrooms are crowded now. When the screen is extended, it would cover more than half of the blackboard, and sometimes even to block the speaker. In this case, it is difficult for those students located in a corner of the classroom to clearly hear the sound coming out of the speaker. Besides, they may suffer from echo, fuzzy, blunt sound or some other problems [9]; secondly, the audio equipment in most multimedia classrooms is bulky, not easy to carry, and featured by the single fixed pattern of sound, the failure of additional audio features to meet the desired effect, and long-term accumulation of dust on the equipment next to the computer which would affect the acoustic effect [10].

Therefore, to solve the aforementioned problems, this essay started from the status quo of multimedia technology and physical education, designed a small and portable multimedia teaching system with special acoustic effects which can shape colorful, changeable stage lighting effects when loading and playing music; furthermore, the audio device is equipped with a dust cover, a Bluetooth connection port, a USB port, a power connector and a computer interface, and diverse fixed patterns, and could be connected to a computer more conveniently. Through conducting experimental application of this system in the course of aerobics, this study provided a reference for application of multimedia teaching system, development of audio and other equipment, and teaching practices of aerobics. 


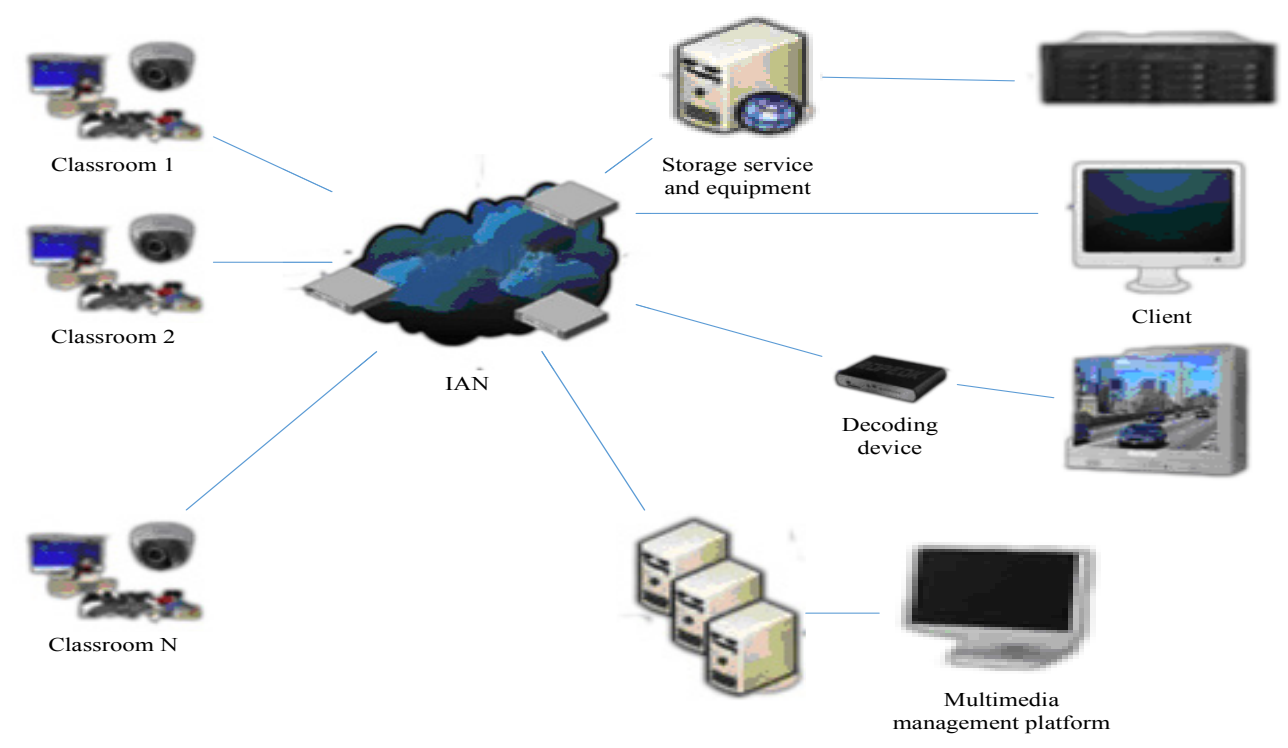

Figure 1. Organizational architecture of the system

\section{ANALYSIS OF THE MULTIMEDIA TEACHING SYSTEM WITH SPECIAL ACOUSTIC EFFECTS}

\section{A. Analysis of System Requirements}

Since the multimedia teaching system with special acoustic effects is mainly used in teaching of aerobics and other physical courses, the system should target at the characteristics of aerobics and realize better effect of sound and lighting, innovative layout templates, expansion of system functions and other features. The system is supposed to not only complete the auxiliary functions in course teaching, but also play a role in curriculum assessment and after-school expansion. The feature of this system in lighting and acoustic effects complies with the requirements of physical teaching. Noticeably, operations of the system include effect display, choice of the template, system update and other acts. Among them, effect display and choice of the template are the core functional requirements which can be achieved by the audio equipment with special acoustic effects. Organizational architecture of the system is demonstrated in Figure 1.

\section{B. Analysis of development technology}

The multimedia teaching system with special acoustic effects was designed based on $\mathrm{B} / \mathrm{S}$ architecture, with the ASP.NET technology as the core technology. The SQL Server 2005 database technology and Microsoft Visual Studio 2008 technology are combined in the design process, while CSS and PHP are employed in the program in the program compilation. The server control architecture developed from the ASP.NET technology covers six interior objects, including Application, Session, ViewState, Request, Response, and Server. Through setting up codes, controls, and temporary files, it can generate multiple page instructions, and then form a complete front-end interface via these page instructions.

\section{DESIGN OF THE MULTIMEDIA TEACHING SYSTEM WITH SPECIAL ACOUSTIC EFFECTS}

\section{A. Design of System Hardware}

Hardware foundation for the multimedia teaching system with special acoustic effects is a portable

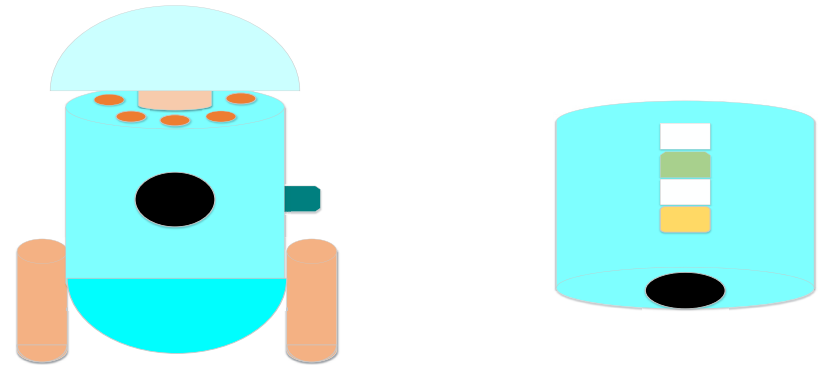

Figure 2. Diagram of the audio equipment with special effects

changeable acoustics available to computers. The audio equipment contains the acoustics itself, control switches, and external interfaces. The inner groove of the acoustics itself is connected with a connecting rod, at the bottom of which there is a fixed hemispherical clump weight with the same diameter of the acoustics itself. The top exterior of the acoustics itself is evenly distributed with 6-15 colored LED lights. Meanwhile, the top of the acoustics itself is connected to the dust cover via a support. Control switches are located in the front of the central part. Along the central back of the acoustics itself from top to bottom, there is a Bluetooth connection port, a USB port, a power connector and a computer interface.

Diagram of the audio equipment with special effects is shown in Figure 2.

As shown in the figure, there are respectively LED lights, a support, a dust cover, the acoustics itself, a pipe buckle, a PA board, a speaker, ear seats, a strut, anti-skid sets, the control switch, a clump weight, a Bluetooth connection port, a USB port, a power connector and a computer interface.

Compared with the existing audio equipment, the portable changeable computer-used acoustics can enhance entertainment, reinforce music aesthetics, offer fixed patterns which tend to be diverse, and can be easily connected with the outside. This audio equipment characterized by more diverse patterns can make use of the clump weight and strut to support the acoustics itself; LED lights can emit bright beams, produce rich lighting effects after the beams are refracted from the reflective 
material at the dust cover's inner wall, and thereby achieve full demonstration of multimedia effects. The PA board in the acoustics body can amplify the sound from the speaker, maintain a better sound effect, and meet the needs of teaching. In order to keep the system open, the acoustics itself can be connected to an external device via the Bluetooth port, USB port and computer interface, to achieve update of internal resources and expansion of function; after the acoustics is connected to an external device, music in the external device can be played in the acoustics.

\section{B. Design of operating system}

The special acoustics design of this study uses embedded operating system, referred to as EOS. Embedded system is an application-centric system based on computer technology which allows reduction of hardware and software and is suitable for dedicated computers of which the application system has requirements on functionality, reliability, cost, size and power consumption [11]. Embedded system generally consists of an embedded processor, related support hardware and an embedded software system, becoming a "device" which is composed of hardware and software and can work independently. The Linux embedded operating system mainly consists of three parts: the kernel, service programs and applications. Its structure is shown in Figure 3.

As demonstrated in Figure 3 shows, the Linux embedded operating system, application for the audio equipment with special effects in this study includes:

(1) Kernel

The kernel is the core program to control the embedded system, and the interface between abstract resources and specific hardware. It implements memory management, file management, as well as program running and scheduling run. At the same time, it is responsible for receiving and sending data packets via the network, accepting user commands and executing accordingly. Efficiency and stability of the kernel program directly affect operation of the entire system.

(2) Service programs

Service programs allow interoperation between applications and the kernel, receive commands from the user and send them to the kernel for execution. These programs include the Shell Command Interpreter, the system for connecting graphical users to the window, kernel programming interface and other system programs.

\section{(3) Applications}

Applications are interfaces for the user to interact with the embedded system, and can be divided into two major categories: utilities and user programs. Utilities provide the user with commonly used commands or functions, including editing, filter and interactive program, while user programs are written to accomplish specific functions.

\section{Advanced Bootloader of the Linux embedded operating system}

The program launcher, Bootloader in this study [12] involves two stages in the starting process, namely: stage 1 and stage 2. Stage 1 is usually implemented in assembly language. Flowchart of the operation of Bootloader is shown in Figure 4.

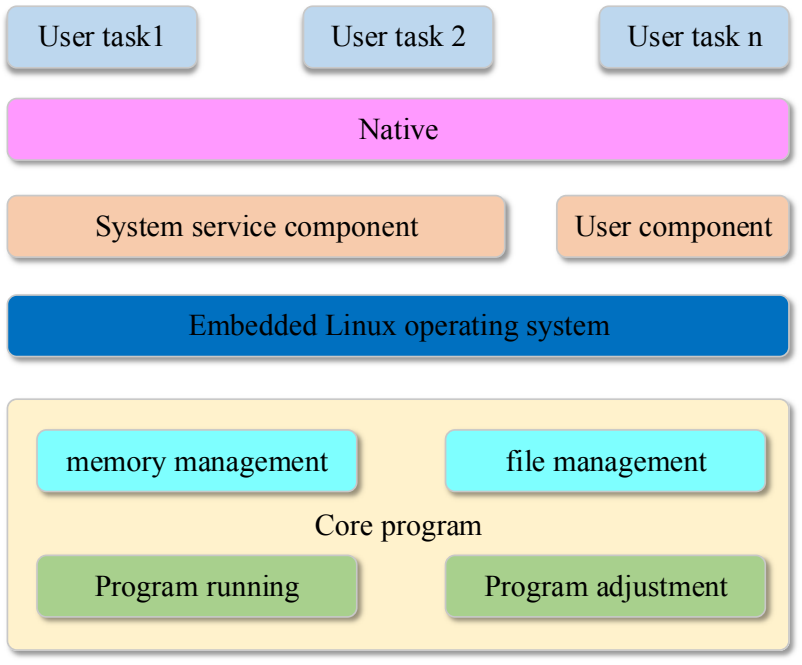

Figure 3. Diagram of the Linux embedded operating system

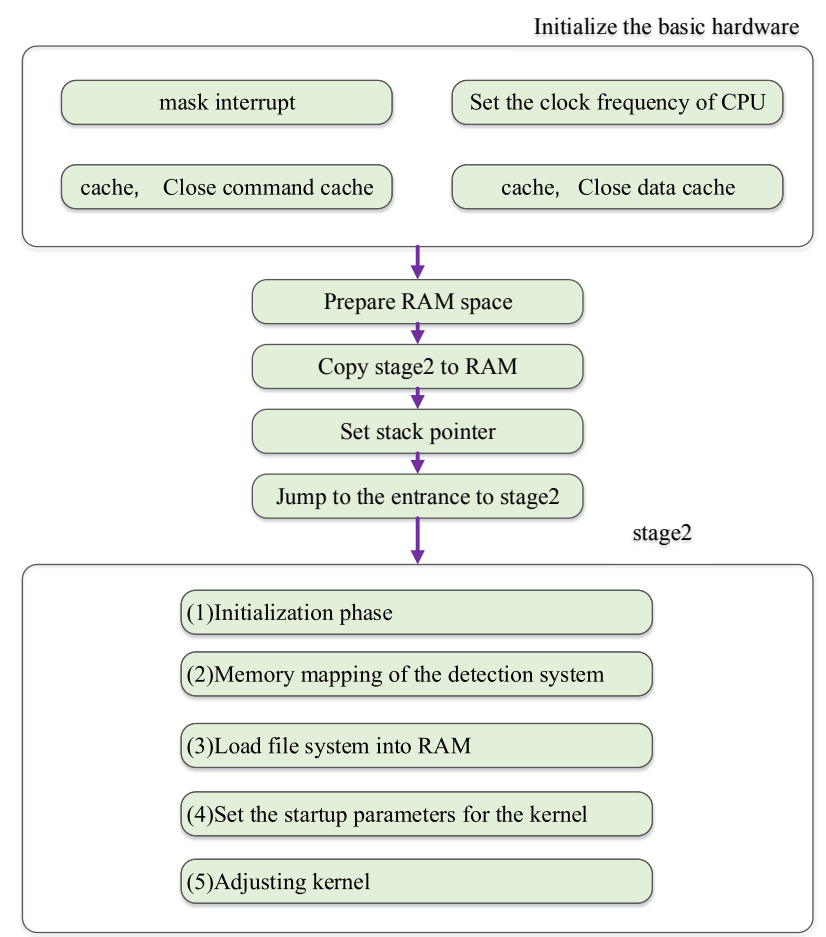

Figure 4. Flowchart of the operation of Bootloader

It can be seen from Figure 4 that stage 1 and stage 2 for Bootloader's starting process respectively include: (1) Initializing basic hardware devices, including inhibiting interrupt, setting the CPU clock frequency, closing the instruction cache and data cache. (2) Preparing RAM space for loading stage 2. (3) Copying stage 2 into RAM;

(4) Setting the stack pointer. (5) Jumping to the beginning of stage 2 .

Stage 2 is usually implemented in C language in the following operations: (1) Initializing hardware devices to be used in this stage, including initializing the serial port, timer and so on. (2) Detecting memory map of the system. (3) Loading the kernel image and root file system into RAM. (4) Setting the kernel boot parameters. (5) Using the kernel. 


\section{INTERFACE DESIGN OF THE PORTABLE AUDIO MULTIMEDIA SYSTEM}

The window interface control module controls terminal playing software of the entire network-based audio system, connections of the server, downloading of files, music playing and other functions.

\section{A. System Programming}

Some programs of the system are as follows:

\section{(1) Headers:}

$<$ minigui/minigui. $\mathrm{h}>, \quad<$ minigui/common. $\mathrm{h}>$ and $<$ minigui/window. $\mathrm{h}>$ are the headers that should be included in all MiniGUI applications. minigui. $\mathrm{h}$ contains definition of the global and universal function to access E1 and some miscellaneous functions; eommon. $h$ involves definition of macros and data types commonly used in MiniGUI; and window. h defines window-related macros, data types, data structures and window functions.

Use of GDI functions and MiniGUl application of controls must also include two other commonly used headers: $<$ minigui/gdi. 1l $>$ and $<$ minigui/contr01. $\mathrm{h}>$.

(2) Program entry

The entry point for a $\mathrm{C}$ program is the main function, and the entry point for a MiniGUI program is MiniGUIMain-i11tMiniGUIMain. The main function has already been defined in MiniGUI, and therefore can use the MiniGUIMain function during the initialization of some of MiniGUl. Therefore, the entry point of each MiniGUI application (no matter server-side mginit or client application) is the MiniGUIMain function.

\section{B. Interface design module}

The interface design module mainly completes design of the system login interface and application interface, including design of the main window, design of the configuration window, design of the menu, etc. Interface design is finished by function call with high efficiency.

(1) Innovative layout template

Information Name: Innovative layout template

Information Data Type: struct

Information Format: struct

Information Source: interface design template

Information Description: Innovative layout template on which establishment of the main window relies

(2) Handle of information disseminated to the window Information Name: Handle of information disseminated to the window

Information Data Type: int

Information Format: HWND

Information Source: interface design templates

Information Description: Handle of information disseminated to the window

(3) Handle of the created main window

Information Name: Handle of the created main window Information Data Type: int

Information Format: HWND

Information Source: interface design templates

Information Description: Handle of the main window created by the main function

(4) Description of main functions:
CreateMainWindowIndirect(): This function is the main window function;

Createmenu(): This function is the function to create menu;

Create-play-coolbar(): This function is the function for setting playing controls and can be used to create playing controls;

InitMp3ConfigDlgt(): This function is the function to create configuration interface. After the window is created, InitMp3ConfigDlgt() can be used to generate configuration interface;

InitConfigDialogProc: This function is the function to set the window for configuration interface. By configuring the window procedure function, InitConfigDialogProc can configure and save network parameters.

Interface and display effects of the system are shown in Figure 5 and Figure 6.

\section{SPECIFIC APPLICATION OF THE MULTIMEDIA TEACHING SYSTEM WITH SPECIAL ACOUSTIC}

In this study, aerobics with a total of 36 course hours was used as the experimental course. The experimental targets included students of an institute of physical education who were admitted in 2013-2015 in different majors. The classes of aerobics were taught by the same teacher responsible for teaching and research based on the use of the multimedia teaching system with special acoustic effects. Students participating in the experiment were from a total of 11 four classes majoring in social sports, sports training, sports health care and physical education. Notably, there was no significant difference between each class.

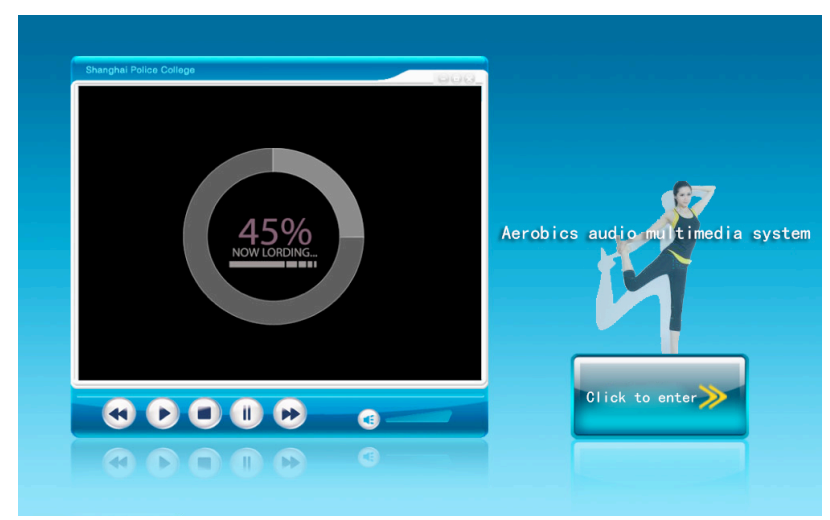

Figure 5. Interface of the multimedia teaching system with special acoustic effects

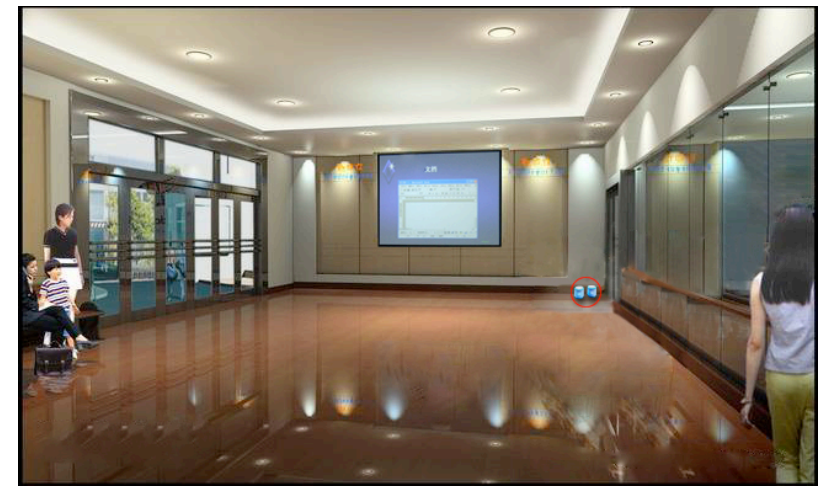

Figure 6. Application interface of the multimedia teaching system with special acoustic effects 
PAPER

A Multimedia Teaching System with Special Acoustic EfFects in the Course of Aerobics

TABLE I.

STATISTICS OF SOCIAL SPORTS AND SPORTS TRAINING

\begin{tabular}{|c|c|c|c|c|c|c|c|c|c|c|}
\hline \multirow{2}{*}{ Index } & \multirow{2}{*}{$\begin{array}{c}\text { Major } / \\
\text { Class }\end{array}$} & \multicolumn{5}{|c|}{ Social sports } & \multicolumn{4}{|c|}{ Sports training } \\
\hline & & \begin{tabular}{|l|} 
Class 1 \\
\end{tabular} & Class 2 & Class 3 & Standard deviation & Variation coefficient & Class 1 & Class 2 & Standard deviation & Variation coefficient \\
\hline \multirow{3}{*}{ Average result } & 2013 & 85.00 & 80.61 & 81.68 & 2.29 & 0.028 & 78.83 & 72.44 & 4.52 & 0.083 \\
\hline & 2014 & 76.23 & 72.89 & 81.47 & 4.33 & 0.056 & 78.81 & 79.30 & 0.35 & 0.004 \\
\hline & 2015 & 76.44 & 78.71 & 80.38 & 1.98 & 0.025 & 84.56 & 84.97 & 0.03 & 0.003 \\
\hline \multirow{3}{*}{ Pass rate $(\%)$} & 2013 & 100 & 92.86 & 100 & 4.12 & 0.042 & 100 & 81.25 & 13.26 & 0.146 \\
\hline & 2014 & 96.67 & 89.29 & 96.67 & 4.25 & 0.045 & 87.88 & 93.75 & 4.15 & 0.046 \\
\hline & 2015 & 89.66 & 96.43 & 92.53 & 3.40 & 0.037 & 97.22 & 96.87 & 0.25 & 0.003 \\
\hline
\end{tabular}

TABLE II.

STATISTICS OF SPORTS HEALTH CARE AND PHYSICAL EDUCATION

\begin{tabular}{|c|c|c|c|c|c|c|c|c|c|c|c|}
\hline \multirow[b]{2}{*}{ Index } & \multirow{2}{*}{$\begin{array}{c}\text { Major/ } \\
\text { class }\end{array}$} & \multicolumn{6}{|c|}{ Sports health care } & \multicolumn{4}{|c|}{ Physical education } \\
\hline & & Class 1 & Class 2 & Class 3 & Class 4 & $\begin{array}{l}\text { Standard } \\
\text { deviation }\end{array}$ & $\begin{array}{l}\text { Variation } \\
\text { coefficient }\end{array}$ & Class 1 & Class 2 & Standard deviation & Variation coefficient \\
\hline \multirow{3}{*}{$\begin{array}{l}\text { Average } \\
\text { result }\end{array}$} & 2013 & 83.10 & 73.97 & 80.77 & 72.26 & 5.22 & 0.067 & 70.36 & 62.53 & 5.54 & 0.083 \\
\hline & 2014 & 83.18 & 72.70 & 75.65 & 72.40 & 5.05 & 0.066 & 78.83 & 75.41 & 2.42 & 0.031 \\
\hline & 2015 & 69.28 & 77.90 & 74.81 & 76.60 & 3.80 & 0.051 & 78.00 & 81.00 & 2.12 & 0.027 \\
\hline \multirow{3}{*}{ Pass rate $(\%)$} & 2013 & 100 & 80.65 & 96.77 & 83.87 & 9.50 & 0.105 & 93.94 & 67.65 & 18.59 & 0.230 \\
\hline & 2014 & 90.91 & 90.00 & 93.75 & 86.68 & 2.91 & 0.032 & 89.66 & 86.21 & 2.44 & 0.028 \\
\hline & 2015 & 87.10 & 93.33 & 90.32 & 92.00 & 2.69 & 0.030 & 93.94 & 90.91 & 2.14 & 0.023 \\
\hline
\end{tabular}

Students' overall achievements in the course of aerobics of included result of the final exam $(70 \%)$ and result of aerobics $(30 \%)$. Questions in the final exam were designed by the Teaching and Research Department and corresponding answers were evaluated in accordance with the same standards. After evaluation of the final exam, the researchers conducted a review and entered students' achievements into EXCEL tables. And then, in accordance with their major and grade, students were divided into different groups, with class as the unit. (See tables I and II)

From the tables above, it can be known that among the 11 classes of 4 majors, only the classes admitted in 2014 in the major of social sports did not have obvious teaching effect, standard deviation and variation coefficient of the average result and passing rate of were reduced at different magnitudes among all the surveyed classes admitted in 2013-2015 in sports training, sports health care and physical education. This result shows that the application of the multimedia teaching system with special acoustic effects in the course of aerobics plays a significant role in enhancing the effect. The reason why the classes admitted in 2014 in the major of social sports obtained an adverse effect may be closely related to the huge amount of social practice tasks for these classes. Students might put a lot of time in social practice, resulting in a significant slowdown in the teaching effect.

As suggested by the statistical data, the multimedia teaching system with special acoustic effects can effectively alleviate the problem of poor expression of audio equipment in the current physical education, and contribute to movement demonstration and innovative choreography of the aerobics course. The multimedia teaching system with special acoustic effects could mobilize students' emotion and attention from multiple angles, while arousing students' interest in aerobics. The gap between students in achievement can be narrowed and the quality of teaching can be improved obviously due to the system.

\section{CONCLUSIONS}

In conclusion, the study found that the rational use of multimedia technology and effective equipment in aerobics teaching is quite helpful in teaching activities. The application of the multimedia teaching system with special acoustic effects in aerobics effectively cultivates students' interest in the course and significantly improves the teaching quality. Giving full paly of the audio equipment with special acoustic effects is of great significance in creating a desired show atmosphere and producing a specific situation. Therefore, teachers should continue to explore new teaching models, have the courage to apply new technology and equipment to classroom practice, and thereby develop a more comprehensive multimedia teaching method which has obvious advantages when compared with the traditional teaching model.

\section{REFERENCES}

[1] Armenteros, M., Liaw, S.S., Fernández, M., Díaz, R.F., \& Sánchez, R.A., "Surveying FIFA instructors' behavioral intention toward the Multimedia Teaching Materials," Computers \& Education, vol. 61, pp. 91-104, February 2013. http://dx.doi.org/10.1016/j.compedu.2012.09.010

[2] Liu, G.H., Meng, Q.G., Yu, H.T., Jiang, N., Terigele, "Research on Status and Countermeasures of Multimedia Classroom Application in Universities of Inner Mongolia," Journal of Inner Mongolia University for Nationalities(Natural Sciences), vol. 90, no. 2, pp. 84-94, April 2015.

[3] Berk, R.A., "Multimedia teaching with video clips: TV, movies, YouTube, and mtvU in the college classroom," International Journal of Technology in Teaching and Learning, vol. 5, no. 1, 1pp. 21, June 2009.

[4] Salajan, F.D., \& Mount, G.J., "Leveraging the power of Web 2.0 tools: a Wiki platform as a multimedia teaching and learning environment in dental education," Journal of dental education, vol. 76, no. 4, pp. 427-436, April 2012.

[5] Shiddiqi, A.M., Ijtihadie, R.M., Emerson, E.Z., Pratomo, B.A. Ahmad, T., Wibisono, W., "Interactive Whiteboard and Instant Quiz for Multimedia Classroom Activity," Iptek Journal of Proceedings, no. 1, pp. 258-263, January 2015. 
PAPER

A Multimedia Teaching System with Special Acoustic EfFects in the Course of Aerobics

[6] Yan, B., "Physical Education Based on Sports Multimedia Technology," Sports World: Academic Edition, no. 9, pp. 74-75, September 2010.

[7] Wang, H.Y., “Aerobics Teaching Reform of Regular Institutions of Higher Learning," Value Engineering, vol. 30, no. 20, pp. 174175, October 2011.

[8] Li, Y., "On the Effect of Applying Audio Mixer in Live Performances," Appreciation, no. 12, pp. 29-29, December 2014.

[9] Metze, F., Ding, D., Younessian, E., \& Hauptmann, A., "Beyond audio and video retrieval: Topic-oriented multimedia summarization," International Journal of Multimedia Information Retrieval, vol. 2, no. 2, pp. 131-144, June 2013. http://dx.doi.org/10.1007/ s13735-012-0028-y

[10] Rinaldi, A.M., "A multimedia ontology model based on linguistic properties and audio-visual features," Information Sciences, vol. 277, pp. 234-246, September 2014. http://dx.doi.org/10.1016/j.ins. 2014.02.017
[11] Berthier, N., Maraninchi, F., Mounier, L., "Synchronous programming of device drivers for global resource control in embedded operating systems," Acm Transactions on Embedded Computing Systems, vol. 12, no. 1s, pp. 81-90, March 2013. http://dx.doi.org/10.1145/2435227.2435235

[12] Wang Y.G., "Analysis and Transplant of Embedded Bootloader Mechanism," Computer Engineering, vol. 36, no. 6, pp. 267-269, March 2010.

\section{AUTHORS}

Yingbao ZHOU is a Lecturer of School of Department of Physical Education, Nanjing Xiaozhuang University, Nanjing 210028, China. His research interests include Physical Education and Sports Exercise. (e-mail: zhouyb111@yeah.net)

Submitted 23 September 2016. Published as resubmitted by the authors 14 October 2016. 